\title{
The Presenilins and Alzheimer Disease
}

\author{
G. Lévesque ${ }^{* \dagger}$, Ph.D., P. St George-Hyslop ${ }^{\dagger}$, M.D., F.R.C.P.(C).
}

\section{INTRODUCTION}

Recent work in the current authors' laboratory and others' has pinpointed various mutated genes associated with early onset forms of familial Alzheimer Disease (FAD). Most recently, mutations in the presenilin 1 (PS1) and presenilin 2 (PS2) genes, located on chromosomes 14 and 1 respectively, have been found to be associated with a large number of families with particularly aggressive early onset FAD. These findings will undoubtedly increase our understanding of the complex pathological processes of both FAD and the more common late onset Alzheimer Disease (AD).

$\mathrm{AD}$ is the most common form of degenerative dementia of the human central nervous system. It is defined clinically by a progressive loss of cognitive function and by the onset of a slowly progressive impairment of memory during mid- to late-adult life (1). The neuropathological hallmarks of this disease included amyloid- $\beta$ peptide $(A \beta)$ deposits (known as senile plaques), neurofibrillary tangles, astrocytic gliosis, and reductions in the number of neurons and synapses in many areas of the brain, but especially from the cerebral cortex and the hippocampus (2).

The overall clinical and pathological phenotypes associated with the early onset autosomal dominant forms of FAD overlap with, and are indistinguishable from, those associated with sporadic AD, with the obvious exception of a younger age-of-onset, a vertical transmission as an autosomal dominant trait, and a slightly more rapid course of the disease in the FADlinked subtypes $(3,4)$. Currently, there are no pathological characteristics pathognomonic of early onset FAD in general or of specific genetic subtypes. It has been hypothesised that the general uniformity of the

* To whom correspondence should be addressed.

$\dagger$ Tanz Neuroscience Building., 6, Queen's Park Crescent, Toronto, Ontario, Canada, M5S 1A8 disease phenotype reflects a common biochemical pathway in which pathological accumulation of the $A \beta$ peptide plays a central role. If so, it would make FAD an inherited syndrome analogous to some metabolic diseases. It is also conceivable that subtle distinguishing differences in the disease phenotype may become apparent once a more robust etiologically based nosologic classification of $\mathrm{AD}$ comes to fruition, and that these may reflect different disease mechanisms that share the same final general phenotype.

The etiology of AD is complex. Multiple epidemiological studies have proposed a number of potential risk factors, including environmental (e.g., head trauma), sociological (e.g., low level of education, depression), biological (e.g., increasing age, Down syndrome, hyperthyroidism, late maternal age) and family history (Down syndrome, AD) (5). Furthermore, several groups have identified apolipoprotein-E (apo-E) genotype as a highly significant modulator of late onset sporadic and familial AD: the relative risk of developing late-onset $\mathrm{AD}$ is increased in individuals homozygotic for the apo-E4 allele (6). However, these individuals do not inevitably develop the disease (7). The variety of AD risk factors testify to the complicated pathogenic process of $\mathrm{AD}$.

The repeated observation in multiple epidemiological surveys that a positive family history is a strong risk factor for $\mathrm{AD}$ clearly suggest that genetic factors play a role in this disease. A simple autosomal dominant mode of inheritance with age dependent penetrance has been observed in many pedigrees, especially those with early age of onset. However, in many families multiply affected by $\mathrm{AD}$, the pattern of inheritance is unclear and could fit one of several genetic models including a high frequency but low penetrance single gene disorder, or a multifactorial model in which several genes and/or nongenetic factors interact (8).

Genetic linkage studies using large numbers of 


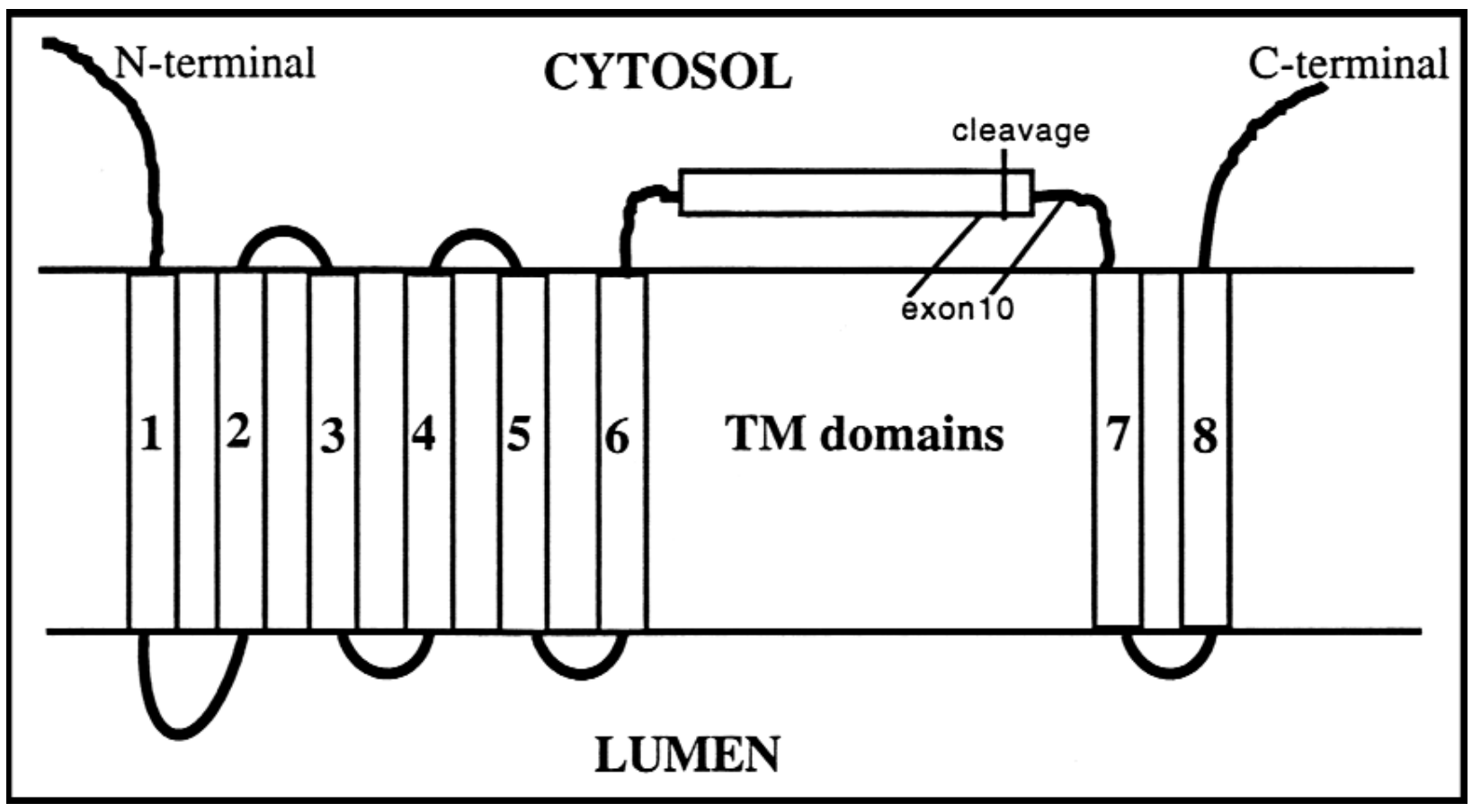

Figure 1. Putative structure of the presenilins.

pedigrees with early-onset autosomal dominant FAD have led to the identification of a series of polymorphic genetic markers located on chromosome 14q24.3 (D14S43, D14S71, D14S77) which showed evidence of linkage to an early onset form of FAD $(9,10,11)$. Subsequent positional cloning studies led to the isolation of a novel gene termed PS1 (12). This Alzheimer susceptibility locus (AD3) is associated with a particularly aggressive form of early-onset (between 30-65 years of age) and accounts for up to 50\% of earlyonset FAD cases. Following the isolation of the PS1 gene causing the AD3 subtype of FAD, another gene associated with FAD (subsequently termed PS2), mapping to chromosome 1, was discovered on the basis of its strong nucleotide and amino acid sequence homology to PS1 $(67 \%$ overall identity and approximately $90 \%$ identity within the transmembrane (TM) domains) (13).

\section{THE PRESENILINS' GENES AND PROTEINS}

The PS1 gene is highly conserved in evolution, being present in C.elegans (14) and D. melanogaster (15), and is transcribed in many different cell types, both within the central nervous system and in peripheral tissues (12). The PS1 promoter contains TATA and CAAT sequences as well as multiple STAT consensus elements that are involved in transcriptional activation following signal transduction (16). The transcript undergoes alternate splicing and analysis of multiple transcripts from brain reveal that the majority of the transcripts begin in Exon 1. A small proportion of transcripts in non-neural tissues contain Exon 2 as the initial transcribed sequence. Both types of transcripts are spliced to Exon 3 as the second of the 13 exons. Exon 4 contains the ATG start codon and shows alternate use of a splice site at the 3' end which results in the variable presence of 12 base pairs encoding 4 amino acids (VRSQ) (17). Another alternate splicing occurs in leucocytes and results in the loss of Exon 9, allowing Exon 10 to reconstitute the hydrophobic domain following TM6. This splicing event is predicted to significantly alter the structure and thus the functional properties of the large cytoplasmic loop following TM6 (Figure 1).

The PS1 protein is predicted to be an integral membrane protein with an open reading frame of 467 amino acids. The protein includes at least 6 hydrophobic membrane spanning domains (TM domains) and two acidic hydrophilic domains located at the N-terminus and after TM6 (Figure 1)(18). Another model suggests 8 TM domains however, data supporting the $8 \mathrm{TM}$ model is in fact more compatible with a 6 TM model (19).

The PS2 gene has an open reading frame of 448 amino acids and the structural organization of its protein is very similar to that of PS1 (13). The pattern of transcription of PS2 is less homogeneous in both brain and in peripheral tissues, where it is maximally expressed in cardiac muscle, skeletal muscle, and pancreas (13). The genomic organization of the PS2 is 
identical to PS1 (20). These observations suggest that the PS1 and the PS2 genes are members of a gene family derived from a common ancestral gene.

\section{PS MUTATIONS AND FAD}

Mutational analysis of the PS1 gene has identified at least 44 different mutations (Table 1), typically located in residues which are highly conserved in evolution, thereby underscoring their likely biological significance. In contrast to the frequency of PS1 mutations, screening of a large data set reveal that PS2 mutations are likely to be rare (21). Mutational analysis of the PS2 gene led to the discovery of two different mutations: one in a pedigree of Italian origin, the other in a group of related families of Volga German ancestry (Table 1). All of the presenilin mutations described thus far are missense mutations with the exception of a PS1 Exon 10 splicing mutation which preserves the open reading frame (other authors have labeled this exon as ${ }^{3}$ Exon 9) (22). To date, in PS1 or PS2, there have been no identified nonsense or frameshift mutations that can leaded to an absence of the protein. This observation has led to the speculation that the effect of these presenilin mutations is a gain of function (23), a gain of dysfunction, or less likely an inhibition of the normal function through a dominant-negative mechanism (24).

The phenotype of these PS2 mutants has a later onset (between 40 and 85 years old) than that associated with mutations in PS1 (onset 35-65 years old). Interestingly, the apo-E genotype has little effect on the age of onset in PS1 and PS2 mutations, suggesting that other modifier loci probably account for much of this variation (21).

\section{PRESENILIN PROTEIN PROCESSING}

It is hoped that an understanding of the cell biology of the PS proteins - notably the differences between wild type and mutated isoforms - will lead to a greater appreciation of their contribution to FAD. Analysis of PS1 metabolism in vivo and in cultured cells such as fibroblasts has revealed that the protein undergoes endoproteolytic cleavage $(25,26)$; the PS2 protein is also endoproteolytically cleaved (27). PS1 cleavage leaves little full-length protein and the predominate products that accumulate are the approximately $28 \mathrm{kDa}$ $\mathrm{N}$-terminal and $18 \mathrm{kDa}$ C-terminal fragments (28). Both of these fragments accumulate to saturable levels and at 1:1 stoichiometry (29). The PS1 FAD-mutant which lacks Exon 10 (residues 290-319) is not subject to endoproteolysis; hence it is clear that the proteolytic event is not necessary for the development of $\mathrm{AD}$. However, it is currently unclear whether the holoprotein, the endoproteolytic fragments, or both, have biological function (28).

\section{CELLULAR LOCALIZATION}

Immunohistochemical studies in mammalian brain suggest that the PS1 protein is predominantly expressed in neurons (30) and in glia (31). In the cell, PS1 is located within intracellular membranes in the endoplasmic reticulum, perinuclear envelope, the Golgi apparatus and some as-of-yet uncharacterized intracytoplasmic vesicles (32,33). Another report suggests that PS1 and PS2 are also localized to the nuclear membrane, its associated interphase kinetochores and the centrosomes, all subcellular structures involved in the cell cycle regulation and mitosis (34). The analysis of the topology of the PS1 protein suggests that the $\mathrm{N}$-terminus, the hydrophilic loop following TM6 and the $\mathrm{C}$-terminus are all located in the cytoplasm $(18,19)$.

\section{PUTATIVE FUNCTIONS FOR THE PRESENILINS}

To investigated the role of PS1 in mammalian development, mice with targeted null mutations (knockout) in the murine homologue of PS1 were generated. These knockout mice developed a deformation of the caudal axial skeleton, hemorrhages in the central nervous system, impairment in neurogenesis, and usually did not survive beyond the first day after birth $(35,36)$. These studies show that PS1 is required for proper formation of the axial skeleton and is involved in normal neurogenesis and survival of progenitor cells and neurons in specific brain subregions (ventricular and subventricular zones in the ventrolateral region, subcortical region of the temporal lobe, lateral ventricles and the dentate gyrus) (36).

The structural and amino acid sequence homologies between PS1 and PS2 suggest that they are likely to have similar or overlapping biological activities. To date, various potential functions have been suggested, including intracellular signaling, suppression of apoptosis and participation in protein/membrane trafficking. However, the precise function(s) of the presenilins are as-of-yet not fully understood. One clue regarding potential function is the weak amino acid sequence homology shown by PS1 to the $C$. elegans SPE-4 protein. SPE-4 is thought to be involved in membrane-budding and fusion events in a membranebound cytoplasmic organelle derived from the Golgi network in spermatocytes (37). This has led to the suggestion that PS1 might have a similar role in protein and membrane trafficking (12). Indeed, its predicted topology suggests hydrophobic association and its cellular distribution confirms its association with various cellular membranes, notably the endoplasmic reticulum.

By searching for suppressors or enhancers of a LIN12 mutation, another C.elegans homologue of PS1 was 
Table 1. Missense mutations in the presenilin genes causing familial Alzheimer Disease. ${ }^{\mathrm{a}}$

\begin{tabular}{|c|c|c|c|}
\hline Codon & Location & Mutation & Phenotype \\
\hline \multicolumn{4}{|c|}{ Presenilin I } \\
\hline 79 & N-terminus loop & Ala -> Val & FAD, onset 64 years \\
\hline 82 & TM1 & Val -> Leu & FAD, onset 55 years \\
\hline 96 & TM1 & Val -> Phe & FAD \\
\hline 115 & TM1 -> TM2 loop & Tyr $->$ His & FAD, onset 37 years \\
\hline 115 & TM1 -> TM2 loop & Tyr -> Cys & FAD \\
\hline 120 & TM1 -> TM2 loop & Glu -> Asp & FAD, onset 48 years \\
\hline 120 & TM1 -> TM2 loop & Glu -> Lys & FAD \\
\hline 135 & TM2 & Asn $\rightarrow$ Asp & FAD \\
\hline 139 & TM2 & Met $->$ Thr & FAD, onset 49 years \\
\hline 139 & TM2 & Met -> Val & FAD, onset 40 years \\
\hline 143 & TM2 & Ile $->$ Thr & FAD, onset 35 years \\
\hline 143 & TM2 & Ile -> Phe & FAD \\
\hline 146 & TM2 & Met $->$ Leu & FAD, onset 45 years \\
\hline 146 & TM2 & Met -> Val & FAD, onset 38 years \\
\hline 146 & TM2 & Met $->$ Ile & FAD, onset 40 years \\
\hline 163 & TM3 interface & His $->$ Arg & FAD, onset 50 years \\
\hline 163 & TM3 interface & His -> Tyr & FAD, onset 47 years \\
\hline 171 & TM3 & Leu $->$ Pro & FAD, onset 40 years \\
\hline 209 & TM4 interface & Gly -> Val & FAD \\
\hline 213 & TM4 & Ile $->$ Thr & FAD \\
\hline 231 & TM5 & Ala $->$ Thr & FAD, onset 52 years \\
\hline 231 & TM5 & Ala -> Val & FAD \\
\hline 233 & TM5 & Met $->$ Thr & FAD, onset 35 years \\
\hline 235 & TM5 & Leu -> Pro & FAD, onset 32 years \\
\hline 246 & TM6 & Ala -> Glu & FAD, onset 55 years \\
\hline 250 & TM6 & Leu -> Ser & FAD \\
\hline 260 & TM6 & Ala -> Val & FAD, onset 40 years \\
\hline 263 & TM6 -> TM7 loop & Cys -> Arg & FAD, onset 47 years \\
\hline 264 & TM6 -> TM7 loop & Pro -> Leu & FAD, onset 45 years \\
\hline 267 & TM6 -> TM7 loop & Pro -> Ser & FAD, onset 35 years \\
\hline 269 & TM6 -> TM7 loop & Arg -> Gly & FAD \\
\hline 269 & TM6 -> TM7 loop & Arg $->$ His & FAD \\
\hline 280 & TM6 -> TM7 loop & Glu -> Ala & FAD, onset 47 years \\
\hline 280 & TM6 -> TM7 loop & Glu -> Gly & FAD, onset 42 years \\
\hline 285 & TM6 -> TM7 loop & Ala -> Val & FAD, onset 50 years \\
\hline 286 & TM6 -> TM7 loop & Leu -> Val & FAD, onset 50 years \\
\hline 290 & TM6 -> TM7 loop & Ser -> Cys & FAD \\
\hline $290-319$ & TM6 -> TM7 loop & Deletion & FAD \\
\hline 318 & TM6 -> TM7 loop & Glu -> Gly & FAD \\
\hline 384 & TM7 & Gly -> Ala & FAD, onset 35 years \\
\hline 392 & TM7 & Leu -> Val & FAD, onset $25-40$ years \\
\hline 410 & TM8 & Cys -> Tyr & FAD, onset 48 years \\
\hline 426 & TM8 & Ala -> Pro & FAD \\
\hline 436 & C-terminal loop & Pro $->$ Ser & FAD \\
\hline \multicolumn{4}{|c|}{ Presenilin II } \\
\hline 141 & TM2 & Asn $->$ Ile & FAD (Volga German), onset $50-65$ years \\
\hline 239 & TM5 & Met -> Val & FAD (Florence), onset variable \\
\hline
\end{tabular}

a Adapted and updated from Lévesque, Yu and St George-Hyslop (53) FAD: familial Alzheimer Disease; TM: transmembrane.

discovered. This protein, named SEL-12, displays about $50 \%$ amino acid sequence identity to PS1. Many FAD mutations of PS1 occur at residues that are conserved in SEL-12 (38). SEL-12 facilitates signaling mechanisms in the LIN-12/Notch family of intercellular receptors which are responsible for intercellular signal transduction specifying the cell fate. Loss of function mutations in SEL-12 result in a defective egg-laying (Egl) phenotype characterized by the accumulation of eggs in the body of the parent nematode (14). The Egl phenotype is also caused by reducing LIN-12 activity, suggesting that SEL-12 is involved either in LIN-12 signaling or in receptor transport or recycling (14). Wild-type human PS1 
expressed from the SEL-12 promoter can rescue the SEL-12 mutant, but mutant PS1 has a strongly decreased rescuing activity (38). These data suggest that PS1 may have a role in the vertebrate Notch signaling pathway. This is further support by the fact that PS1 knockout mice show developmental abnormalities similar to mice with targeted knockouts of the murine Notch1 gene $(35,39)$.

\section{PRESENILIN AND AB}

Several lines of evidence strongly suggest that $A B$ (derived from amyloid precursor protein (APP) processing) deposition in brain, and particularly deposition of the neurotoxic 42 amino acid $A B$ isoform (Aß42), has a central role in the pathogenesis of $\mathrm{AD}$ (40). Recently, it was demonstrated that fibroblasts cultured from PS1 and PS2 mutant carriers secreted elevated amounts of AB42 (41). Additionally, the authors' group has created a series of human cell lines in which different wild-type or mutant PS1/PS2 cDNAs were expressed. All of the presenilin mutant transfected cells secreted significantly increased levels of Aß42 compared to wild type controls (42). These observations suggest a dominant gain of function for the presenilin mutations because even though the endogenous wildtype alleles are present, the level of Aß42 is increased in the cells expressing mutant cDNAs. How mutant presenilins alter APP processing is unknown. It is proposed that PS1 is involved as a regulatory cofactor in the proteolytic cleavage of the carboxy-terminal fragments of APP (mediated by gamma-secretase) after their generation by amino-terminal cleaving alpha- and beta-secretases (43). The mutations in PS1 presumably lead to increased cleavage of APP carboxy-terminal releasing extra $\mathrm{A} \beta 42$ (44).

Several independent lines of transgenic mice have been created in which wild-type or mutant presenilins have been over-expressed in mouse brain. All of the mutant, but none of the wild-type mice, show an increase in the production of Aß42 in brain $(42,45)$. The same phenomena was observed in transgenic mice over-expressing mutant APP, suggesting a common pathological pathway by which the presenilins and BAPP mutations cause AD (41). Nevertheless, despite the Aß42 accumulation in the brain of the mutant PS1 and BAPP transgenic mice, at the present time, these mice have not shown any neuron death, but synapse loss has been reported in APP transgenics. The PS1 mutant transgenic mice do not show abnormal neuropathology; however, these mice showed reduced spontaneous alternation performance in a $\mathrm{Y}$ maze. The breeding of the mutant APP (K670L+ M671L) transgenic line with a mutant PS1(M146L) transgenic result in a doubly transgenic progeny who develop a higher numbers of
$\mathrm{A} \beta$ deposit in cerebral cortex and hippocampus suggesting that PS1 and APP mutation are synergistic (46).

\section{PRESENILINS AND APOPTOSIS}

Apoptosis is a biological process involved in the normal development and homeostasis of different cell types (47). Dysregulation in this process may be involved in neurodegenerative disorders. It has been reported that mutant PS2 induces apoptosis in cultured nerve cells under conditions in which normal PS2 has no effect (48). Furthermore, the expression of a truncated form of PS2 (ALG3) in T-cell hybridoms blocks apoptosis induced by the Fas pathway by interfering with the activity and/or activation of Caspase-3, a key component of the apoptotic pathway $(49,50)$. During apoptosis, both PS1 and PS2 were shown to be cleaved by a caspase- 3 family protease independently of the normal endoproteolytic cleavage. It was suggested that the fragments produced by this cleavage may serve as pro-apoptotic effectors that make cells more sensitive to apoptosis (51). However, another group reported that PS2, but not PS1, is cleaved by caspase-3 (52). Thus, the exact contribution of presenilin to apoptosis in the neuropathology of $\mathrm{AD}$ remains unclear and further experiments are necessary to assign a role for presenilins in the induction of apoptosis in the development of AD.

\section{CONCLUDING REMARKS}

Beyond their contribution to early onset FAD, it is still unknown whether the presenilins have a role in the majority of late onset AD. Although PS1 and PS2 are expressed at very low levels in the brain, conservative missense substitutions in these proteins cause an aggressive early onset form of AD. These observations imply that they are involved in a very important biological pathway that, when disregulated, leads to AD. Identifying the biochemical intermediates in the presenilins' pathway will be very important in identifying the function of these proteins and will help to find the causes of early onset and possibly late-onset $\mathrm{AD}$ cases. These finding will then lead in the development of rational therapeutic strategies against this devastating neurodegenerative disease.

\section{ACKNOWLEDGMENTS}

Research in the authors' laboratory is support by the Medical Research Council of Canada, the Alzheimer Association of Ontario, the Canadian Genetic Diseases Network, the EJLB Foundation, the Alzheimer Society of Canada (GL) and the Howard Hughes Medical Research Foundation. 


\section{REFERENCES}

1. Kertesz A, Mobs RC. In: Gauthier S, ed. Clinical Diagnosis and Management of Alzheimer's Disease. Martin Dunitz; 1996.

2. St George-Hyslop P. Genetic of Alzheimer Disease. In: Markesbery W. ed. Neuropathology of dementing diseases. London, Edward Arnold Publishers; 1997.

3. Foncin JF, Salmon D, Supino-Verbo V, et al. Alzheimer's presenile dementia transmitted in an extended kindred. Revue Neurologique (Paris) 141(3):194-202; 1985.

4. Goudsmit J, White BJ, Weitkamp LR, et al. Familial Alzheimer's disease in two kindreds of the same geographic and ethnic origin: a clinical and genetic study. Journal of Neurological Sciences 49(1): 78-89; 1981.

5. Katzman R, Kawas CH. The Epidemiology of Dementia and Alzheimer Disease. In: Terry RD, Katzman R, Bick KL, eds. Alzheimer Disease. New York, NY: Raven Press; 1994.

6. Roses AD. Apolipoprotein $\mathrm{E}$ alleles as risk factors in Alzheimer's disease. Annual Review of Medicine 47:387-400; 1996.

7. Lambert JC, Perez-Tur J, Dupire MJ, et al. Distortion of allelic expression of apolipoprotein E in Alzheimer's disease. Human Molecular Genetics 6(12): 2151-2154; 1997.

8. Farrer LA, Myers RH, Cupples LA, St George-Hyslop PH, Bird $\mathrm{TD}$, Rossor MN. Transmission and age-at-onset patterns in familial Alzheimer's disease: Evidence for heterogeneity. Neurology 40(3 pt 1): 395-403; 1990.

9. Schellenberg GD, Bird TD, Wijsman EM, et al Genetic linkage evidence for a familial Alzheimer's disease locus on chromosome 14. Science 258(5082): 668-671; 1992.

10. St George-Hyslop P, Haines J, et al. Genetic evidence for a novel familial Alzheimer's disease locus on chromosome 14. Nature Genetics 2(4): 330-334; 1992.

11. Van Broeckhoven C, Backhovens H, Cruts M, et al. Mapping of a gene predisposing to early-onset Alzheimer's disease to chromosome 14q24.3. Nature Genetics 2(4): 335-339; 1992.

12. Sherrington R, Rogaev EI, Liang Y, et al. Cloning of a gene bearing missense mutations in early-onset familial Alzheimer's Disease. Nature 375(6534): 754-760; 1995.

13. Rogaev EI, Sherrington R, Rogaeva EA, et al. Familial Alzheimer's Disease in kindreds with missense mutations in a gene on chromosome 1 related to the Alzheimer's Disease type 3 gene. Nature 376(6543): 775-778; 1995.

14. Levitan D, Greenwald I. Facilitation of lin-12 mediated signalling by sel-12, a Caenorhabditis elegans S182 Alzheimer's disease gene. Nature 377(6547): 351-354; 1995.

15. Boulianne GL, Livne-Bar I, Humphreys JM, et al. Cloning and characterization of the Drosophila presenilin homologue. Neuroreport 8(4): 1025-1029; 1997.

16. Rogaev EI, Sherrington R, Wu C, et al. Analysis of the 5' sequence, genomic structure, and alternative splicing of the presenilin-1 gene (PSEN1) associated with early onset Alzheimer disease. Genomics 40(3): 415-424; 1997.

17. The Alzheimer's disease Collaborative Group: The structure of presenilin 1(S182) gene and the identification of six novel mutations in early onset AD families. Nature Genetics 11(2): 219-222; 1995

18. Lehmann S, Chiesa R, Harris DA. Evidence for sixtransmembrane domain structure of presenilin 1. Journal of Biological. Chemistry 272(18): 12047-12051; 1997.

19. Doan A, Thinakaran G, Borchelt G, et al. Protein topology of presenilin 1. Neuron 17(5): 1023-1030; 1996.

20. Levy-Lahad E, Poorkaj P, Wang K, et al. Genomic structure and expression of STM2, the chromosome 1 familial Alzheimer disease gene. Genomics 34(2):198-204; 1996.
21. Sherrington R, Froelich S, Sorbi S, et al. Alzheimer's disease associated with mutations in presenilin 2 is rare and variably penetrant. Human Molecular Genetics 5(7): 985-988; 1996.

22. Perez-Tur J, Froelich S, Prihar G, et al. A mutation in Alzheimer's disease destroying a splice acceptor site in the presenilin-1 gene. Neuroreport 7(1): 297-301; 1995.

23. Van Broeckhoven C. Presenilins and Alzheimer disease. Nature Genetics 11(3): 230-232; 1995.

24. Hutton M, Hardy J. The presenilins and Alzheimer's disease. Human Molecular Genetics 6(10): 1639-1646; 1997.

25. St George-Hyslop PH, Lévesque G, Lévesque L, et al. Two homologous genes causing early-onset familial Alzheimer's disease. Cold Spring Harbor Symposia on Quantitative Biology Vol. LXI: 559-564; 1996.

26. Thinakaran G, Borchelt DR, Lee MK, et al. Endoproteolysis of presenilin 1 and accumulation of processed derivatives in vivo. Neuron 17(1): 181-190; 1996.

27. Kim TW, Pettingell WH, Hallmark OG, et al. Endoproteolytic cleavage and proteasomal degradation of presenilin 2 in transfected cells Journal of Biological Chemistry 272(17):11006-11010; 1997.

28. Podlisny MB, Citron M, Amarante P, et al. Presenilin proteins undergo heterogeneous endoproteolysis between Thr291 and Ala299 and occur as stable N- and C- terminal fragments in normal and Alzheimer brain tissue. Neurobiology of Disease 3(4): 325-337; 1997.

29. Thinakaran G, Harris CL, Ratovitski T, et al. Evidence that levels of presenilins (PS1 and PS2) are coordinately regulated by competition for limiting cellular factors. Journal of Biological Chemistry 272: 28415-28422; 1997.

30. Kovacs DM, Fausett HJ, Page KJ, et al. Alzheimer-associated presenilins 1 and 2: Neuronal expression in brain and localization to intracellular membranes in mammalian cells. Nature Medicine 2(2): 224-229; 1996.

31. Lah JJ, Heilman CJ, Nash NR, et al. Light and electron microscopic localization of presenilin 1 in the primate brain. Journal of Neuroscience. 17(6): 1971-1980; 1997.

32. De Strooper B, Beullen M, Contreras B, et al. Phosphorylation, subcellular localization, and membrane orientation of the Alzheimer's disease-associated presenilins. Journal of Biological Chemistry 272(6): 3590-3598; 1997.

33. Walter J, Capell A, Grunberg J, et al. The Alzheimer's diseaseassociated presenilins are differentially phosphorylated proteins located predominantly within the endoplasmic reticulum. Molecular. Medicine. 2(6): 673-691; 1996.

34. Li J, Xu M, Zhou H, Ma J, Potter H. Alzheimer presenilins in the nuclear membrane, interphase kinetochores, and centrosomes suggest a role in chromosome segregation. Cell 90(5): 917-927; 1997.

35. Wong PC, Zheng $\mathrm{H}$, Chen $\mathrm{H}$, et al. Presenilin 1 is required for Notch 1 and DII 1 expression in the paraxial mesoderm. Nature 387(6630): 288-292; 1997.

36. Shen J, Bronson RT, Chen DF, Xia W, Selkoe DJ, Tonegawa S. Skeletal and CNS defects in presenilin-1 deficient mice. Cell 89(4): 629-639; 1997.

37. L'Hernault SW, Arduengo PM. Mutation of a putative sperm membrane protein in Caenorhabditis elegans prevents sperm differentiation but not its associated meiotic divisions. Journal of Cellular Biology 119(1): 55-68; 1992.

38. Baumeister R, Leimer U, Zweckbronner I, Constanze J, Grunberg J, Haass C. Human presenilin-1, but not familial Alzheimer's disease (FAD) mutants, facilitate Caenorhabditis elegans Notch signalling independently of proteolytic processing. Genes Function 1: 149-159; 1997.

39. Conlon RA, Reaume AG, Rossant J. Notch 1 is required for the 
coordinate segmentation of somites. Development 121(5): 1533-1545; 1995.

40. Selkoe DJ. Amyloid beta-protein and the genetics of Alzheimer's disease. Journal of Biological Chemistry 271(31): 18295-18298; 1996.

41. Scheuner D, Eckman C, Jensen M, et al. Secreted amyloid betaprotein similar to that in the senile plaques of Alzheimer's disease is increased in vivo by the presenilin 1 and 2 and APP mutations linked to familial Alzheimer's disease. Nature Medicine 2(8): 864-870; 1996.

42. Citron M, Westaway D, Weiming X, et al. Mutant Presenilins of Alzheimer's disease increase production of 42-residue amyloid B-protein in both transfected cells and transgenic mice. Nature Medicine 3(1): 67-72; 1997.

43. DeStrooper B, Saftig P, Craessaerts K, et al. Deficiency of presenilin-1 inhibits the normal cleavage of amyloid precursor protein. Nature 391(6665): 387-390; 1998.

44. Haas C., Selkoe D.J. A technical KO of amyloid-B peptide. Nature 391(6665): 339-340; 1998.

45. Duff K, Eckman C, Zehr C, et al. Increase amyloid-beta42(43) in brains of mice expressing mutant presenilin 1. Nature 383(6602): 710-713; 1996.

47. Kerr JFR, Wyllie AH, Currie AR. Apoptosis: a basic biological phenomenon with wide-ranging implications in tissue kinetics. British Journal of Cancer 26(4): 239-257; 1972.

48. Wolozin B, Iwasaki K, Vito P, et al. Participation of presenilin2 in apoptosis: enhanced basal activity conferred by an Alzheimer mutation. Science 274(5293): 1710-1713; 1996.

49. Vito P, Lacana E, D'Adamio L. Interfering with apoptosis: $\mathrm{Ca}(2+)$-binding protein ALG-2 and Alzheimer's disease gene ALG-3. Science 271(5248): 521-525; 1996.

50. Lacana E, Ganjei JK, VitoP, D'Adamio L. Dissociation of apoptosis and activation of IL-1ß-converting enzyme/Ced-3 proteases by ALG-2 and the truncated Alzheimer's disease gene ALG-3. J. Immunology 158(11): 5129-5135; 1997.

51. Kim TW, Petingell WH, Jung YK, Kovacs DM, Tanzi RE. Alternative cleavage of Alzheimer-associated presenilins during apoptosis by a caspase-3 family protease. Science 277: $373-376$; 1997.

52. Vito P, Ghayur T, D'Adamio L. Generation of anti-apoptotic presenilin-2 polypeptides by alternative transcription, proteolysis, and caspase-3 cleavage. Journal Biological Chemistry 272: 28315-28320; 1997.

53. Lévesque G, Yu G, St George-Hyslop PH. Genetics of Alzheimer disease. Current Neurology 17: 137-156; 1997.

Dr. G. Levesque received his B.Sc. in Biochemistry from the University of Quebec at Montreal (Montreal, Quebec, Canada) and his Ph.D. in Cellular and Molecular Biology from Laval University (Quebec, Quebec, Canada). He is currently pursuing post-doctoral studies at the University of Toronto Centre for Research in Neurodegenerative Diseases (Toronto, Ontario, Canada) under the supervision of Dr. P. St George-Hyslop. Dr. Levesque was awarded the first Memorial Frist/Jus prize for research in neurodegenerative diseases and the Post-Doctoral award from the Alzheimer Society of Canada. Dr. P. St. George-Hyslop is the discoverer of the presenilin genes associated with aggressive early onset forms of familial Alzheimer disease. He received his M.D. degree from the University of Ottawa (Ottawa, Ontario, Canada) and then undertook post-graduate training in Internal Medicine and in Neurology at the University of Toronto (Toronto, Ontario, Canada). He then pursued a fellowship in Molecular Genetics in the Neurogenetics Laboratory at Massachusetts General (Boston, Massachusetts, USA) and subsequently became an instructor in Neurology at Harvard Medical School (Boston, Massachusetts, USA) and the Massachusetts General Hospital Hospital before returning to the University of Toronto. He is currently professor in the Department of Medicine, Division of Neurology, at the University of Toronto, and a consultant physician in Neurology at the Toronto Hospital. Dr. St George-Hyslop has been awarded the Francis McNaughton prize of the Canadian Neurological Society, the Potamkin from the American Academy of Neurology, the Metropolitan Life Award, the gold medal from the Royal College of Physicians, the Michael Smith Award for Excellence, the Medical Research Council of Canada Scholar Award, and the Medical Research Council of Canada Scientist Award. 